\title{
Dendroclimatologia na Amazônia: aplicações e potencialidades
}

\author{
Dendroclimatology in the Amazon basin: applications and potentialities \\ José Guilherme Roquette ${ }^{\mathrm{I}}$, Francisco de Almeida Lobo ${ }^{\mathrm{II}}$, Leone Francisco Amorim Curado ${ }^{\mathrm{III}}$
}

\begin{abstract}
Resumo
Os efeitos de mudanças climáticas na Amazônia podem ser estudados por meio da dendroclimatologia, a partir da análise das relações entre as variáveis climáticas e meteorológicas e o crescimento do xilema de espécies arbóreas. O xilema constitui um verdadeiro arquivo natural porque permite o registro dessas variáveis na madeira, o que pode ser de grande utilidade para a compreensão dos impactos potenciais que as atividades antrópicas podem exercer sobre o clima. O objetivo deste artigo é abordar aspectos inerentes ao estado da arte sobre os métodos dendroclimatológicos e sua aplicação nas florestas tropicais da Amazônia para análise da formação de anéis de crescimento em plantas lenhosas. A revisão sobre o tema foi realizada em diversas bases bibliográficas, tanto clássicas como mais recentes, e foi constatado que, embora seja uma ciência antiga, ainda são poucos os estudos dendroclimatológicos conduzidos na Amazônia. As florestas são ecossistemas sensíveis e estudos relacionados aos efeitos que podem sofrer em virtude de mudanças climáticas são relevantes para o planejamento socioeconômico e o desenvolvimento de políticas públicas. Palavras-chave: Dendrocronologia; Mudanças climáticas; Bacia Amazônica; Anéis de crescimento
\end{abstract}

\begin{abstract}
The effects of climatic changes in the Amazon basin can be studied through dendroclimatology, from the analysis of the relations between climatic and meteorological variables and the growth of the xylem of arboreal species. The xylem constitutes a true natural file because it allows the recording of these variables in the wood, which can be very useful for understanding the potential impacts that anthropic activities may have on the climate. The objective of this article is to discuss aspects inherent to the state of the art on dendroclimatic methods and their application in the tropical forests of the Amazon region for analysis of the formation of growth rings in woody plants. The review on the subject was carried out in several bibliographic bases, both classic and more recent, and it was found that, although it is an ancient science, there are still few dendroclimatic studies conducted in the Amazon basin. Forests are sensitive ecosystems and studies related to the effects they may suffer from climate change are relevant to socioeconomic planning and the development of public policies.
\end{abstract}

Keywords: Dendrochronology; Climatic changes; Amazon basin; Growth rings

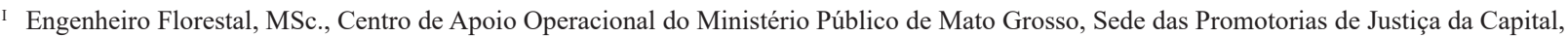
Av. Des. Milton Figueiredo Ferreira Mendes, s/n, Centro Político e Administrativo, CEP 78049-928, Cuiabá (MT), Brasil. guilhermeroquette@ yahoo.com.br (ORCID: 0000-0003-0645-9173)

II Agrônomo, Dr., Professor do Programa de Pós-graduação em Física Ambiental, Universidade Federal de Mato Grosso, Av. Fernando Corrêa, 2367, Boa Esperança, CEP 78060-900, Cuiabá (MT), Brasil. fdealobo@gmail.com (0000-0002-5670-0351)

III Físico, Dr., Professor do Programa de Pós-graduação em Física Ambiental, Universidade Federal de Mato Grosso, Av. Fernando Corrêa, 2367, Boa Esperança, CEP 78060-900, Cuiabá (MT), Brasil. leonecurado@gmail.com (0000-0002-0375-7725)
} 


\section{Introdução}

A Amazônia, o maior bioma em extensão do mundo, ocupa cerca de 2/5 da América do Sul, o que equivale a $5 \%$ da superfície terrestre (aproximadamente 6,5 milhões de $\mathrm{km}^{2}$ ), e contempla diversos ecossistemas que abrigam a maior biodiversidade existente (IBGE, 2004). As formações vegetais predominantes são florestas, denominadas de florestas tropicais (tropical rainforests) na classificação fitogeográfica geral, que são encontradas nas zonas de clima quente, úmido e de elevada precipitação pluvial, como nas regiões das Antilhas, África, Ásia, Insulíndia e Américas do Sul e Central (SOARES, 1953).

A característica principal do clima amazônico é a convecção tropical, que é regulada por vários fenômenos de circulação atmosférica, principalmente aqueles de grande escala, o que confere à região pelo menos três subclimas diferentes, de acordo com a classificação de Köppen (Af: equatorial chuvoso; Am: monção tropical; Aw: tropical seco e úmido). Especialmente devido à extensão da floresta tropical, a Amazônia influencia o clima em grande escala, ao mesmo tempo que é influenciada por ele, possuindo grande importância em nível global (ALMEIDA et al., 2017).

De acordo com Schöngart e Junk (2007), na Bacia Amazônica e em outras regiões tropicais, os ecossistemas e meios socioeconômicos podem ser mais afetados devido à vulnerabilidade a eventos climáticos extremos, impulsionados pelo aumento das emissões de gases do efeito estufa e crescimento populacional.

Para melhor compreender os efeitos das mudanças climáticas sobre as florestas tropicais, as informações registradas na madeira ao longo do crescimento e desenvolvimento das árvores podem ser utilizadas como um arquivo natural para a reconstrução de climas passados (HUGHES, 2002). Os anéis de crescimento formados nas árvores são especialmente adequados para essa análise porque apresentam datação confiável, resolução anual, ampla replicação, longevidade acima de milhares de anos, ampla representação entre espécies e microssítios, bem como sensibilidade às condições climáticas (SHEPPARD, 2010).

A datação de anéis de crescimento em plantas lenhosas é conhecida como dendrocronologia, ciência à qual pertence a dendroclimatologia, uma subdisciplina que se preocupa com a associação entre os anéis de crescimento e o clima, utilizando modelos estatísticos (FRITTS, 1971). De acordo com Sheppard (2010), é mais comum a reconstrução da precipitação ou da temperatura, que são analisadas pela frequência em anos extremos, variações nas condições médias, intervalos de variabilidade a longo prazo e mudanças na variabilidade interanual. No entanto, existem outras aplicações dendroclimáticas para a avaliação e análise de anomalias climáticas passadas e de circulações atmosféricas, que controlam o clima, relacionando as variações da largura dos anéis com variáveis meteorológicas, como pressão, índices de circulação e frequências de tipos de circulação (FRITTS, 1971).

Poucos estudos dendroclimatológicos foram desenvolvidos na região Amazônica, o que dificulta o reconhecimento das interações existentes entre o crescimento vegetal e o clima em nível regional, assim como a percepção dos impactos em um cenário de mudanças climáticas. A integração de estudos dendroclimatológicos realizados em diferentes regiões da Amazônia e com diferentes espécies pode corroborar a reconstrução e prospecção do clima e seus efeitos sobre a vegetação da Amazônia e auxilar na tomada de decisão no desenvolvimento de políticas públicas.

Em decorrência disso, o objetivo deste trabalho é abordar os métodos dendroclimatológicos e sua aplicação no estudo da formação dos anéis de crescimento em plantas lenhosas das florestas tropicais da Amazôna. A revisão do tema foi delimitada pela pesquisa de referências, tanto clássicas como mais recentes, nas seguintes bases bibliográficas: Scielo; DOAJ; e Google Acadêmico, bem como na biblioteca virtual Periódicos Capes. Para a busca foram utilizadas as seguintes palavras-chave e expressões (em inglês, português e espanhol): dendrocronologia; dendroclimatologia; anéis de crescimento; Amazônia; Floresta Amazônica; mudanças climáticas. Após o procedimento exploratório, procedeu-se também à consulta analítica das referências utilizadas naquelas obras que foram encontradas nas bases bibliográficas. 


\section{Formação de anéis de crescimento em plantas lenhosas}

Além de fatores intrínsecos à espécie, o crescimento das árvores é afetado pelas condições ambientais, sendo a relação entre os fatores que resultam no crescimento dependente de restrições filogenéticas, da morfologia individual da árvore e da variabilidade climática (FICHTLER; WORBES, 2012). Essa interação entre gradientes biológicos e variáveis ambientais complica a tarefa de modelar suas relações, porque controla as dimensões das camadas de crescimento e a forma como elas variam na árvore (FRITTS, 1971). Apesar da importância, os fatores fisiológicos, genéticos e ambientais que influenciam a vida das células, bem como os seus efeitos combinados, ainda são mal-compreendidos (BRÄKER, 2002).

De acordo com Gartner et al. (2002), muitos fatores ambientais e ecológicos causam efeitos sobre o desenvolvimento da planta. Entre eles, os autores citam o fogo, a posição da copa, as doenças, os efeitos de insetos, as inundações, o vento, a neve, a fertilidade do solo, a disponibilidade de água, a competição entre plantas, a poluição e o clima.

Com relação ao clima, algumas condições estão diretamente relacionadas ao crescimento, enquanto outras são inversamente proporcionais (FRITTS, 1971). Além disso, as espécies possuem diferentes sensibilidades climáticas, apresentando respostas diferentes em termos de crescimento (BONINSEGNA et al., 2009).

O câmbio vascular é o principal meristema lateral responsável pelo crescimento em diâmetro da árvore (crescimento secundário), atividade que é regulada por fatores genéticos e ambientais do sítio (WIMMER, 2002). Durante o crescimento ocorre a divisão celular, o aumento da célula e o espessamento da parede celular, formando estruturas ao longo do tempo que podem ser visualizadas na madeira (GARTNER et a., 2002). A disposição e arranjo dos elementos celulares da madeira, conforme as características distintas formadas com as condições de crescimento, constituem uma fonte de informações sobre o habitat das árvores (FERRERO; VILLALBA; RIVERA, 2014). Assim, a compreensão do mecanismo que impulsiona a dinâmica de produção e desenvolvimento celular, e como isso impacta as estruturas anatômicas, deve permitir melhores resultados nos estudos de climas passados em uma resolução subanual (DOWNES; WIMMER; EVANS, 2002).

A plasticidade das variáveis anatômicas da madeira demonstra como as árvores podem se adaptar às mudanças das condições ambientais, por isso é possível existir diferenças entre e dentro de indivíduos de populações, assim como relacionadas aos sítios em que se densenvolvem (FICHTLER; WORBES, 2012). O contraste entre as células formadas em cada estação do ano é frequentemente utilizado para delinear as fronteiras dos anéis de crescimento, que são camadas formadas no xilema secundário de troncos e ramos (FRITTS, 1971).

Os fatores genéticos determinam a estrutura básica dos anéis das árvores, mas ocorre uma série de modificações causadas pelo ambiente, à qual é dada toda atenção em estudos dendrocronológicos (BRÄKER, 2002; WIMMER, 2002). Tanto a largura da ca|mada no sentido radial como a aparência da estrutura celular podem ser funções de fatores ambientais atuando durante o período de crescimento, bem como funções da hereditariedade potencial da árvore (FRITTS, 1971). De acordo com Tarelkin et al. (2016), os anéis anuais de crescimento variam dentro do indivíduo dependendo do órgão considerado, sendo mais visíveis na madeira do tronco do que na dos galhos. Além disso, também podem ocorrer diferenças na visibilidade dos anéis quando árvores juvenis ou adultas são avaliadas (SOLIZ-GAMBOA et al., 2011).

Mudanças nos fatores climáticos influenciam a iniciação ou cessação da atividade cambial, que conduz à formação do lenho inicial e tardio durante as estações do ano com condições climáticas diferenciadas (FRITTS, 1971). Os limites de um anel de crescimento podem ser identificados a partir de (1) diferenças no diâmetro e distribuição dos vasos do lenho inicial e tardio, (2) ocorrência de fibras de paredes mais espessas e lúmens de menor diâmetro na madeira tardia ou (3) existência de uma faixa marginal de parênquima axial (FERRERO; VILLALBA; RIVERA, 2014). Os anéis de crescimento podem estar, ainda, indistintos ou ausentes na madeira, caraterísticas essas que são altamente variáveis, e suas causas e formas de classificação não são consenso na comunidade científica (TARELKIN et al., 2016).

De acordo com Fritts e Swetnam (1989), a largura de um anel de crescimento é expressa em função 
de seis componentes básicos em uma equação: (1) a tendência de crescimento relacionada à idade no ano "t", que é compartilhada entre espécies em um mesmo sítio; (2) as variações no crescimento relacionadas ao clima, comuns a um grupo de árvores no ano " $t$ ", incluindo a persistência que essas variações significam devido ao pré-condicionamento fisiológico e à interação do clima com outros fatores do sítio; (3) os pulsos de distúrbios endógenos originados da competição, da mudança da estatura da árvore e de outras forças atuantes em árvores específicas durante o ano " $t$ "; (4) os pulsos de distúrbios exógenos originados por forças de fora da comunidade florestal, tais como geadas, incêndios ou infestações de insetos, durante o ano " $t$ "; (5) as variações devido aos poluentes antropogênicos que tenham impacto no crescimento radial da árvore no ano "t"; e (6) as variações aleatórias nos fatores que influenciam o crescimento radial no ano " $t$ " para uma árvore ou raio, incluindo erros na medição do anel de crescimento.

A largura do anel de crescimento diminui com a idade cambial, com o aumento da altura em troncos jovens e com a redução do crescimento apical, existindo, portanto, uma tendência decrescente da largura dos anéis intrínseca a fatores como o envelhecimento e mudanças na geometria do tronco (FRITTS; SWETNAM, 1989). Além disso, em árvores adultas de algumas espécies os anéis de crescimento podem ser identificados com menos dificuldade do que na fase juvenil (SOLIZ-GAMBOA et al., 2011). É preciso considerar ainda que existem espécies em que a largura dos anéis de crescimento é pouco afetada por variações do ambiente (FRITTS, 1971).

De acordo com Hughes (2002), muitos fatores são conhecidos por influenciarem a variabilidade de anéis de crescimento, mas muitas cronologias realizadas têm mostrado fortes correlações lineares com variáveis climáticas, como as médias sazonais de precipitação e temperatura. Assim, como resultado das flutuações interanuais do clima, podem surgir anéis de crescimento claramente visíveis, como ocorre em algumas espécies de regiões com chuvas e inundações sazonais (BENISTON, 2002; BONINSEGNA et al., 2009).

As mudanças ano a ano na largura dos anéis de crescimento da base do tronco estão mais relacionadas a variações macroclimáticas, enquanto as alterações na largura dos anéis em regiões superiores do tronco estão mais relacionadas a variações microclimáticas (FRITTS, 1971). Em florestas tropicais e subtropicais, a homogeneidade relativa da temperatura ao longo do ano, sem períodos de baixas temperaturas, como ocorre em regiões temperadas, facilita uma atividade cambial contínua durante todo $\mathrm{o}$ ano. Entretanto, naquelas regiões tropicais e subtropicais marcadas pela sazonalidade da precipitação, formando estações secas durante o ano ou áreas inundadas durante o período chuvoso, a dormência do câmbio é favorecida, formando, consequentemente, bandas de crescimento sazonal (SCHONGART et al., 2005; FERRERO; VILLALBA; RIVERA, 2014).

Nem todas as plantas lenhosas são capazes de produzir uma sequência de anéis de crescimento datáveis, como é o caso de muitas das espécies arbóreas de regiões tropicais que não apresentam estruturas distintas ou a periodicidade da formação não é anual (BONINSEGNA et al., 2009). Fritts (1971) relata que durante um ano desfavorável ao crescimento, o estímulo cambial, originário das pontas do tronco, pode nunca atingir a base do caule, o que faz com que seja mais provável a ocorrência de falsos anéis anuais de crescimento nas proximidades da copa que em regiões da base do tronco. Soliz-Gamboa et al. (2011), ao estudarem a formação de anéis de crescimento em espécies tropicais, identificaram que em algumas delas há a probabilidade de ocorrência de um anel falso para cada dez anéis anuais de crescimento.

A existência de anéis de crescimento em árvores tropicais foi questionada durante muito tempo (WIMMER, 2002). No entanto, o avanço da dendrocronologia em regiões tropicais tem demonstrado como muitas espécies apresentam a formação de anéis anuais e permitem o desenvolvimento de cronologias (BONINSEGNA et al., 2009). Apesar disso, deve-se sempre ter o cuidado de reconhecer as alterações nas estruturas anatômicas dos anéis de crescimento que podem prejudicar a acurácia da datação e as inferências sobre os fatores relacionados às suas características.

\section{Métodos em estudos dendroclimatológicos}

A grande vantagem da abordagem dendrocronológica é a capacidade de datar os anéis de crescimento de acordo com o ano civil de nosso calendário, com alto grau de confiança (HUGHES, 2002). 
Como toda ciência, a dendroclimatologia se baseia em alguns princípios básicos, descritos por Fritts (1971), para que seja possível a utilização dos anéis de crescimento para reconstrução de clima passado:

(1) Princípio do fator limitante - cada processo é sempre limitado por algum fator ou conjunto de fatores que podem surgir de condições externas ou internas do organismo. O objetivo da dendroclimatologia é a reconstrução do clima passado a partir de características estruturais de anéis de crescimento, por isso, torna-se importante a utilização de árvores em que o crescimento do anel seja mais ou menos limitado, direta ou indiretamente, por algum fator climático.

(2) Princípio da seleção do sítio - a seleção de amostras deve ocorrer em ambientes em que o crescimento foi altamente limitado por um determinado fator, por isso é considerado uma extensão do princípio do fator limitante. Assim, a variação da largura do anel deve ocorrer de acordo com o fator limitante do sítio e ser comum em muitas árvores, maximizando o "sinal" que representa a variável climática e minimizando "ruídos" originados de outros fatores.

(3) Princípio da sensibilidade e medição da varibilidade de baixa e alta frequência - a variabilidade da largura de um anel para outro fornece um dos melhores indicadores de estresse causado pelo clima. Isso acontece porque frequentemente os fatores climáticos são os mais limitadores do crescimento, sendo as variações nas larguras respostas da variação climática. Assim, uma vez que a sensibilidade média mede as mudanças na largura do anel em relação à largura dos anéis adjacentes, ela é influenciada por mudanças de curto prazo e por variações de alta frequência no clima.

(4) Princípio da datação cruzada - a construção de uma cronologia exige que a variação das caracterísiticas do anel, especialmente a largura, sejam reconhecidas e sincronizadas entre todas as amostras de uma determinada região, fornecendo assim um tipo de controle "experimental". Portanto, entende-se que as flutuações ano a ano das variáveis climáticas produzem variações síncronas na estrutura dos anéis de crescimento das amostras e podem ser datadas de acordo com o nosso calendário civil anual.

(5) Princípio da replicação - a repetição das cronologias derivadas nas árvores é a melhor evidência de que a datação cruzada é real e pode ser utilizada para identificar e reconstruir precisamente um período de crescimento anual, cujos resultados são estimativas razoáveis de algum componente do clima regional. Assim, se o comportamento das variações das larguras dos anéis das amostras for semelhante, a precisão da datação pode ser conseguida com uma pequena quantidade de árvores amostradas. Porém, quanto maior o tamanho da amostra menor o ruído de fatores não climáticos e o crescimento médio de cada ano se aproxima da cronologia climática. Sheppard (2010) relata que cronologias geralmente são bem replicadas quando a amostragem compreende mais que vinte árvores.

(6) Princípio da modelagem da relação ambiental - as variáveis climáticas são combinadas com os anéis de crescimento por meio de modelagens estatísticas, com o objetivo de simular o sistema complexo que limita o crescimento. A modelagem, normalmente realizada por meio de técnicas de regressão, deve retratar as hipóteses físicas e fisiológicas do inter-relacionamento entre as variáveis, que são avaliadas por testes de significância. Quando os relacionamentos entre a estrutura do anel e os fatores climáticos não são conhecidos precisamente, devem-se adotar modelos mais flexíveis do que modelos mais rígidos a priori.

A amostragem é uma etapa importante para resultados consistentes, e deve ser realizada de acordo com os princípios da dendrocronologia, além de considerar o objetivo e as hipóteses do estudo (BRÄKER, 2002). Assim, inicialmente, um estudo dendroclimatológico requer que as espécies arbóreas produzam anéis de crescimento distintos na maioria dos anos, com características que possam ser dendrocronologicamente datadas e que atinjam idades suficientes para abrangerem o tempo de controle necessário para uma investigação particular (FRITTS; SWETNAM, 1989). Os anéis de crescimento têm a geometria de cones empilhados em uma árvore e, por essa razão, quanto mais próximo do solo as amostras forem retiradas maior será o número de anéis amostrados (WILES; CALKIN; JACOBY, 1996).

As amostras podem ser coletadas de forma não destrutiva, com a utilização da sonda de Pressler (ou trado de incremento) para perfuração do tronco e remoção de uma haste de alguns milímetros de diâmetro. No entanto, de acordo com Lopez, Villalba e Bravo (2013), devido à alta densidade de grande parte das madeiras tropicais, o uso dessa ferramenta pode ser inviabilizado. Além disso, a ocorrência de variações no perfil do anel de crescimento e a possibilidade de existência de falsos anéis implica a necessidade de coleta de pelo menos duas hastes por árvore (FRITTS, 1971). 
Outro modo de obtenção de amostras para estudos dendrocronológicos é a coleta oportunista de discos da seção transversal do tronco na base das árvores, em áreas sob desmatamento ou exploração madeireira (FERRERO; VILLALBA; RIVERA, 2014). As seções transversais fornecem um campo maior de observação, facilitam o delineamento dos incrementos anuais de crescimento e são uma opção para regiões tropicais, nas quais existe a dificuldade de perfuração de espécies que produzem madeira de alta densidade (LOPEZ; VILLALBA; BRAVO, 2013).

As cronologias são baseadas, em sua maioria, na largura dos anéis de crescimento, a partir da técnica denominada datação cruzada (FRITTS, 1971; FRITTS; SWETNAM, 1989; HUGHES, 2002). Nesse caso, após a coleta, as amostras (discos de madeira) devem ser trabalhadas com lixas de diferentes gramaturas para que se tenha um bom polimento, que permita a medição da largura dos anéis de crescimento sob um microscópio e um micrótomo deslizante, utilizando-se dendrômetros, como o Lintab ${ }^{\mathrm{TM}}$ ou o Velmex Measuring System (FRITTS, 1971; BRÄKER, 2002; BONINSEGNA et al., 2009; LOPEZ; VILLALBA; BRAVO, 2013), conectados a um computador pessoal.

Mensuradas as larguras dos anéis de crescimento, deve-se calcular os coeficientes de correlação entre a largura dos anéis das séries individuais e a cronologia mestre construída a partir da média das amostras replicadas, para assim verificar a qualidade das cronologias e auxiliar na identificação de anéis ausentes ou falsos (FRITTS, 1971; BRÄKER, 2002; LOPEZ; VILLALBA; BRAVO, 2013; LOPEZ; VILLALBA, 2016). Em seguida, para remover as mudanças na largura dos anéis de crescimento relacionadas com o aumento da idade da árvore e maximizar a porcentagem de variância comum nas variações da largura dos anéis entre amostras da cronologia, deve-se realizar um processo conhecido por padronização, que é feito a partir do ajuste de uma curva de crescimento e a estimação de um índice da largura do anel, desenvolvido a partir da razão entre a série real e a ajustada (Figura 1) (FRITTS, 1971; COOK; PETERS, 1981). A curva de crescimento pode ser adequada por equações de regressão em modelagem autorregressiva, a partir de funções exponenciais, polinomiais, logarítmicas, lineares ou suaves, que representem o crescimento empírico da árvore (FRITTS, 1971; DUNISCH; MONTÓIA; BAUCH, 2003; BONINSEGNA et al., 2009; NEUKOM; GERGIS, 2011; MENDIVELSO et al., 2014; PAREDES-VILLANUEVA et al., 2015).

Figura 1 - Comparação entre séries cronológicas de medidas absolutas (1) e padronizadas (2) das variações nas larguras dos anéis de crescimento das espécies Swietenia macrophylla King (linha espessa) e Cedrela odorata L. (linha fina) em Floresta Amazônica primária em Aripuanã, Mato Grosso, Brasil. Fonte: Dünisch, Montóia e Bauch (2003).

Figure 1 - Comparison between chronological series of absolute measures (1) and standardized (2) variations in growth ring widths of the species Swietenia macrophylla King (dense line) and Cedrela odorata L. (thin line) in the Amazonian primary forest in Aripuanã, Mato Grosso state, Brazil. Source:

Dünisch, Montóia e Bauch (2003).

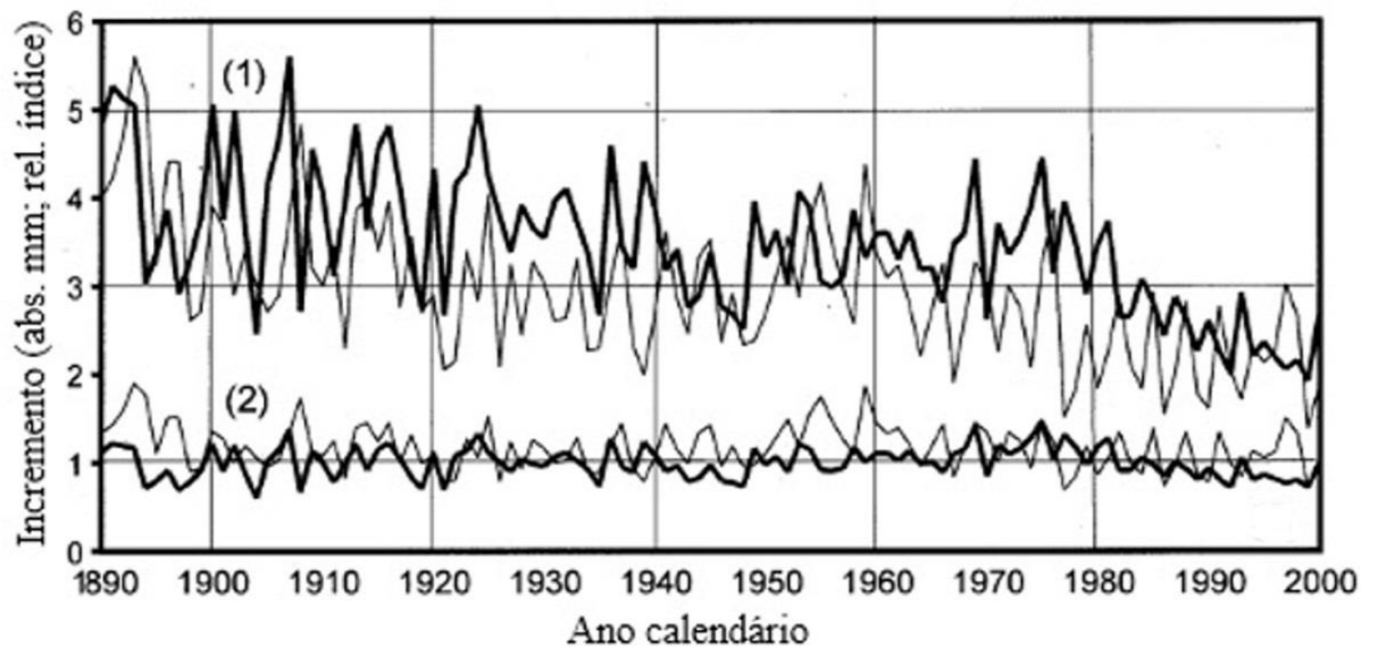

Ci. Fl., Santa Maria, v. 29, n. 1, p. 451-462, jan./mar., 2019 
Outras estatísticas são comumente utilizadas para avaliar a qualidade da cronologia derivada das amostras, incluindo o desvio padrão, a autocorrelação de primeira ordem, a sensibilidade média, que é uma medida da variação relativa de um anel de um ano para o próximo, a RBar (correlação de interséries), que é uma medida do sinal comum nas variações do anel das árvores, e o EPS (sinal expresso da população), que é uma medida do sinal total presente em uma cronologia (por exemplo, um intervalo de cinquenta anos) em comparação com a cronologia das amostras replicadas, e pode indicar a suficiência amostral (consideramse valores do EPS acima de 0,85 como uma amostragem bem replicada) (FRITTS, 1971; BRIFFA, 1995; MENDIVELSO et al., 2014; LOPEZ; VILLALBA, 2016). Uma série de softwares que podem ser utilizados para análises e modelagens em dendrocronologia pode ser consultada em Bräker (2002).

Construída a cronologia de anéis de crescimento, estatisticamente ela pode ser calibrada com condições climáticas sazonais representadas por observações meteorológicas (FRITTS, 1971). A calibração pode ser realizada utilizando técnicas de mínimos quadrados em um modelo de regressão multivariada que estima o valor da cronologia a partir de uma série de variáveis meteorológicas preditoras, como precipitação e temperatura mensais (FRITTS; SWETNAM, 1989).

Existem também outras técnicas para mensurar as propriedades da madeira objetivando a datação, como a densidade dos anéis, o tamanho dos vasos ou a composição isotópica (BONINSEGNA et al., 2009). Para avaliar a natureza anual das bandas de crescimento, diversos estudos adicionais podem ser realizados para confirmação da datação, como o emprego de métodos gráficos para comparação das amostras (Skeleton plots), o uso de radiocarbono e isótopos de oxigênio ou cálcio, além de dados estratigráficos de materiais subfósseis (WILES; CALKIN; JACOBY, 1996; TARELKIN et al., 2016).

$\mathrm{O}$ avanço da tecnologia também tem proporcionado uma série de opções para melhorar o entendimento da formação da madeira e os resultados das pesquisas com anéis de crescimento, como a densitometria de raios- $\mathrm{x}$, a espectometria de fluorescência de raios-x e a espectrometria vibracional de absorção de infravermelho próximo (DOWNES; WIMMER; EVANS, 2002). Tecnologias de digitalização, como tomografia, escaneamento óptico e sônico, também têm sido promissoras para estudos dendrocronológicos (GARTNER et al., 2002).

As informações de climas anteriores dependem de dados meteorológicos obtidos por registros instrumentais, e por esse motivo muitas vezes o intervalo de tempo analisado por dendrocronologia pode ser limitado pela falta de dados (BENISTON, 2002; HUGHES, 2002). Além disso, os dados meteorológicos são obtidos em várias escalas de tempo, como horas, dias, semanas ou meses, no entanto, os anéis de crescimento geralmente se associam ao clima em um período sazonal ou anual (SHEPPARD, 2010). Os efeitos do clima no crescimento das árvores também não são necessariamente verificados no mesmo ano do evento climático, podendo ser necessário considerar variáveis climáticas de uma sequência temporal de períodos retrospectivos ao do ano de formação do anel (FRITTS; LOFGREN; GORDON, 1979).

Pesquisas recentes envolvem dados e modelos de árvores que se concentram na simulação de tendências futuras do crescimento das árvores, com base nos resultados do modelo climático global para estabelecer a evolução futura do clima e avaliar qual será a resposta no crescimento das árvores frente às mudanças climáticas (BENISTON, 2002). Assim, modelos de simulação são necessários em nível de indivíduos, populações e comunidades de árvores para compreender os efeitos combinados das mudanças de precipitação, concentração de $\mathrm{CO}_{2}$, temperatura, nutrientes e crescimento e sobrevivência das árvores, que em muitos casos são pobremente compreendidos (ZUIDEMA et al., 2013).

\section{Aplicações e potencialidades da dendroclimatologia na Amazônia}

Poucos estudos dendroclimatológicos foram desenvolvidos na Amazônia e tinham como objetivo, principalmente, relacionar a largura dos anéis de crescimento com a precipação e a influência do fenômeno El Niño - oscilação Sul sobre as taxas de crescimento de diferentes espécies (DÜNISCH; MONTÓIA; BAUCH, 2003; SCHÖNGART et al., 2004; 2005; BRIENEN; ZUIDEMA, 2005; SOLIZ-GAMBOA et al., 2011; BRIENEN et al., 2012). Outros estudos foram conduzidos para verificar a influência indireta da precipitação sobre a formação de anéis de crescimento devido aos regimes de inundação que são observados em algumas regiões da Amazônia (SCHÖNGART et al., 2002), além da influência da temperatura e pressão de vapor d'água no sinal de isótopos de oxigênio registrados nas bandas de crescimento anual

Ci. Fl., Santa Maria, v. 29, n. 1, p. 451-462, jan./mar., 2019 
(BRIENEN et al., 2012).

A influência indireta do clima sobre o crescimento e a fenologia de árvores na Amazônia venezuelana, devido aos impactos causados por inundações sazonais, foi investigada por Schöngart et al. (2002). Esses pesquisadores verificaram que as inundações ocasionam a perda de parte ou de todas as folhas de diversas espécies, levando à dormêrcia cambial e à formação do anel de crescimento. Assim, nos anos em que o nível das águas é menor, e a fase terrestre maior, há um aumento do incremento na largura dos anéis de crescimento, com exceção das espécies com caules suculentos, para as quais não ficou claro quais os fatores que influenciaram o crescimento radial e a fenologia.

Dünisch, Montóia e Bauch (2003) investigaram o incremento da largura dos anéis de crescimento das espécies Swietenia macrophylla King (Meliaceae) e Cedrela odorata L. (Meliaceae) em Aripuanã, Mato Grosso, município situado na Amazônia meridional. Os autores verificaram uma significativa correlação da largura dos anéis de crescimento de $S$. macrophylla durante todo o período chuvoso, enquanto em $C$. odorata o incremento da largura dos anéis de crescimento foi correlacionado significativamente com a precipitação dos meses de março e maio do período de crescimento anterior. Também observaram que a formação de anéis de crescimento em $S$. macrophylla foi restrita à fase adulta, diferentemente de $C$. odorata, cuja formação dos anéis ocorreu anualmente tanto na madeira juvenil como na adulta.

A influência do El Niño sobre o incremento da largura dos anéis de crescimento também foi estudada por Schöngart et al. $(2004 ; 2005)$ para as espécies Piranhea trifoliata Baill. (Euphorbiaceae) e Macrolobium acaciifolium (Benth.) Benth. (Fabaceae), respectivamente, nas regiões oeste e central da Amazônia. Ambos os estudos foram conduzidos em regiões que sofrem o efeito de inundações sazonais, e observou-se que, em anos de El Niño, devido à anomalia negativa na quantidade de precipitação e, consequentemente, à redução do nível das águas, ocorre um incremento na largura dos anéis de crescimento. De acordo com os resultados apresentados, verificou-se que nos dois últimos séculos a severidade do fenômeno El Niño aumentou significativamente na região.

Brienen e Zuidema (2005) avaliaram a formação de anéis de crescimento de seis espécies da Amazônia boliviana (Amburana sp., Bertholletia sp., Cedrela sp., Cedrelinga sp., Peltogyne sp. e Tachigali sp.) e verificaram correlações da largura dos anéis com a precipitação para quatro delas. Três espécies apresentaram forte correlação entre o crescimento e o início da estação chuvosa, enquanto uma demonstrou ser mais sensível à precipitação no final da estação no crescimento anterior. De acordo com os autores, os resultados da pesquisa demonstraram claramente que a análise de anéis de crescimento pode ser empregada com sucesso nos trópicos e é um método promissor para várias linhas de pesquisa.

Outro estudo que também verificou a formação de anéis de crescimento na madeira juvenil e adulta de espécies da Amazônia boliviana, bem como a correlação do crescimento com a precipitação, foi desenvolvido por Soliz-Gamboa et al. (2011). Os pesquisadores sincronizaram a datação dos anéis de crescimento com a datação por $\mathrm{C}^{14}$ e verificaram que nas espécies estudadas a delimitação dos anéis de crescimento é similar para madeira juvenil e adulta e que eles são formados anualmente. O crescimento de todas as espécies estudadas também apresentou correlação com a precipitação, embora de diferentes formas.

A reconstrução da precipitação na Amazônia também foi desenvolvida por Brienen et al. (2012) com base nos isótopos de oxigênio $\left(\mathrm{O}^{18}\right)$ presentes nos anéis anuais de crescimento de $C$. odorata no norte da Bolívia. O resutado do estudo demonstrou como o sinal do isótopo de oxigênio fica preservado na madeira durante o período chuvoso, com fraca influência da temperatura e pressão de vapor d'água, além de ter forte correlação com os isótopos de oxigênio de amostras de gelo dos Andes. Os autores encontraram também, uma importante influência do El Niño no crescimento das árvores nas décadas mais recentes.

A análise de anéis de crescimento das árvores tem se mostrado essencial para a reconstrução do clima passado em muitas partes do mundo, fornecendo dados para períodos em que a observação instrumental direta não está disponível (BENISTON, 2002). De acordo com Martinelli (2004), a melhoria da qualidade dos dados exige estudos em uma ampla distribuição geográfica, a fim de estabelecer uma rede de cronologias feitas a partir da análise de anéis de crescimento.

A comparação das reconstruções climáticas na região amazônica, com base na análise dos anéis das árvores e no relacionamento com os padrões de circulação atmosférica, é útil para verificar tendências passadas e futuras na mudança do clima global e suas implicações para o desenvolvimento 
socioeconômico e o bem-estar humano (BONINSEGNA et al., 2009). Os aumentos do nível de $\mathrm{CO}_{2}$ na atmosfera, a deposição de nutrientes e as mudanças climáticas, provavelmente, já afetaram o crescimento das árvores e a dinâmica das florestas tropicais (ZUIDEMA et al., 2013). Por outro lado, os impactos de longo prazo das variações dos fenômenos oceânicos-atmosféricos e de outros fenômenos relacionados, tais como incêndios, inundações e infestações de pragas em florestas, estão emergindo em várias regiões da América do Sul, requerendo mais estudos dendroclimatológicos para o entendimento dos efeitos das variações climáticas e suas conexões (BONINSEGNA et al., 2009). A implementação de tais pesquisas ajudaria a aprimorar a compreensão sobre os processos envolvidos na dinâmica das florestas que estão associados à mudança do clima (BENISTON, 2002).

Para preencher essa lacuna de conhecimento em florestas tropicais, Zuidema et al. (2013) argumentam que são necessárias quatro abordagens: (1) a análise de anéis de crescimento; (2) a análise de isótopos estáveis; (3) a validação de modelos mecanísticos; e (4) experimentos controlados de larga escala. Isso porque o desenvolvimento de dendrocronologias em florestas de climas tropicais e subtropicais continua sendo um grande desafio, principalmente devido a problemas relacionados com a visualização dos anéis de crescimento, com a uniformidade circular da seção transversal dos troncos e com a dataçãocruzada (BONINSEGNA et al., 2009). Peters et al. (2015) recomendam a aplicação de múltiplos métodos para auxiliar na análise de tendências de crescimento e melhorar a sensibilidade, confiabilidade e acurácia dos resultados, como, por exemplo, a correção dos incrementos da largura dos anéis com a área transversal e a divisão das amostras por classes de diâmetro. No entanto, embora o número de cronologias na Amazônia ainda seja baixo, seus resultados têm sido úteis nos estudos das relações clima-crescimento, nos quais os fortes "sinais" climáticos observados nos anéis de algumas espécies têm demonstrado como a dendroclimatologia é uma ciência promissora na região.

De acordo com Boninsegna et al. (2009), esse conjunto de dados paleoclimáticos derivados da dendrocronologia pode ser utilizado para validar os modelos climáticos globais, com potencial para alcançar avanços importantes na resolução e qualidade devido à alta precisão das informações. Os autores enfatizam que a capacidade preditiva aprimorada dos modelos climáticos é relevante para o planejamento da gestão de recursos naturais e desenvolvimento de políticas públicas.

A dendrocronologia também é uma ciência poderosa para estudar dinâmicas da regeneração de espécies arbóreas tropicais com falhas de recrutamento, permitindo a identificação dos picos de recrutamento na estrutura populacional e das taxas de crescimento das árvores (VLAM et al., 2014). Com base na distribuição da idade e nas características dos anéis das árvores, inferências podem ser feitas sobre a dinâmica passada da floresta para auxiliar no manejo florestal sustentável na Amazônia (LOPEZ; VILLALBA; BRAVO, 2013; VLAM et al., 2014). De acordo com Fonseca Junior, Piedade e Schöngart (2009), a aplicação de certos critérios no manejo de florestas pode ser inadequado para algumas espécies arbóreas, caso não se tenham informações confiáveis sobre o crescimento e a regeneração natural, podendo ocasionar efeitos negativos na estrutura populacional. Além disso, os dados registrados nas árvores também podem ser utilizados para avaliar em que medida a interferência antropogênica no sistema pode levar a uma taxa e amplitude de mudança significativamente diferente das mudanças no passado, quando o estresse provocado pelo Homem era mínimo (BENISTON, 2002).

\section{Considerações Finais}

Embora seja uma ciência antiga, ainda são poucos os estudos dendroclimatológicos conduzidos na Amazônia, existindo uma lacuna sobre esse conhecimento em diferentes sítios da região. A dúvida que existia sobre a formação de anéis de crescimento em espécies amazônicas foi sanada por alguns estudos que avaliaram as estruturas anatômicas e a influência, direta ou indiretamente, de variáveis climáticas sobre suas formações. Ainda assim, o cenário atual exige que mais estudos sejam realizados com outras espécies e em sítios com condições distintas, a fim de avaliar quais resultados representam melhor a variabilidade climática e podem ser utilizados para a reconstrução do passado e prognose sobre mudanças futuras com maior confiabilidade.

Os processos de seleção do sítio, amostragem, datação e calibração com varíaveis climáticas 
devem ser criteriosamente definidos, seguindo padrões descritos na literatura. A adoção de processos semelhantes para análises dendroclimatológicas permite que diversos estudos realizados em diferentes regiões possam ser comparados, tornando mais precisas as inferências sobre áreas geográficas maiores. Assim, potenciais mudanças sobre o crescimento e desenvolvimento de árvores e comunidades florestais devido às mudanças no clima regional e global poderão ser melhor compreendidas.

A Amazônia, por ser o bioma com maior biodiversidade mundial, apresenta muitas oportunidades para estudos dendroclimatológicos, principalmente devido à alta sensibilidade a fatores ambientais, em especial os climáticos, que algumas espécies apresentam. As florestas da Amazônia são ecossistemas sensíveis, e estudos relacionados aos efeitos que podem sofrer em virtude de mudanças climáticas são relevantes para o planejamento socioeconômico e o desenvolvimento de políticas públicas, visando a proteção e uso sustentável desses ambientes. Os anéis de crescimento em espécies amazônicas representam um arquivo natural sobre o clima, preservado no tronco das árvores, e podem ser de grande utilidade para a compreensão dos impactos potenciais que as atividades antrópicas podem exercer sobre o clima e a sustentabilidade da vida na região.

\section{Referências}

ALMEIDA, C. T. et al. Spatiotemporal rainfall and temperature trends throughout the Brazilian Legal Amazon, 1973-2013. International Journal of Climatology, Reading, v. 37, n. 4, p. 2013-2026, 2017.

BENISTON, M. Climate modeling at various spatial and temporal scales: where can dendrochronology help? Dendrochronologia, Verona, v. 20, n. 1/2, p. 117-131, 2002.

BONINSEGNA, J. A. et al. Dendroclimatological reconstructions in South America: a review. Palaeogeography, Palaeoclimatology, Palaeoecology, Amsterdam, v. 281, n. 3/4, p. 210-228, 2009.

BRÄKER, O. U. Measuring and data processing in tree-ring research - a methodological introduction. Dendrochronologia, Verona, v. 20, n. 1/2, p. 203-216, 2002.

BRIFFA, K. R. Interpreting high-resolution proxy climate data: the example of dendroclimatology. In: VON STORCH, H.; NAVARRA, A. (Ed.). Analysis of climate variability, applications of statistical techniques. Heidelberg: Springer, 1995. p. 77-94.

BRIENEN, R. J. W. et al. Oxygen isotopes in tree rings are a good proxy for Amazon precipitation and El Nino-Southern Oscillation variability. Proceedings of the National Academy of Sciences, Washington, v. 109, n. 42, p. 16957-16962, 2012.

BRIENEN, R. J. W.; ZUIDEMA, P. A. Relating tree growth to rainfall in Bolivian rain forests : a test for six species using tree ring analysis. Oecologia, New York, v. 146, p. 1-12, 2005.

COOK, E. R.; PETERS, K. The smoothing spline: a new approach to standardizing forest interior tree-ring width series for dendroclimatic studies. Tree-ring Bulletin, Tucson, v. 41, p. 45-53, 1981.

DOWNES, G. M.; WIMMER, R.; EVANS, R. Understanding wood formation: gains to commercial forestry through tree-ring research. Dendrochronologia, Verona, v. 20, p. 37-51, 2002.

DÜNISCH, O.; MONTÓIA, V. R.; BAUCH, J. Dendroecological investigations on Swietenia macrophylla King and Cedrela odorata L. (Meliaceae ) in the central Amazon. Trees - Structure and Function, Berlin, v. 17, p. 244-250, 2003.

FERRERO, M. E.; VILLALBA, R.; RIVERA, S. M. An assessment of growth ring identification in subtropical forests from northwestern Argentina. Dendrochronologia, Verona, v. 32, n. 2, p. 113-119, 2014.

FICHTLER, E.; WORBES, M. Wood anatomical variables in tropical trees and their relation to site 
conditions and individual tree morphology. IAWA Journal, Leiden, v. 33, n. 2, p. 119-140, 2012.

FONSECA JÚNIOR, S. F.; PIEDADE, M. T. F.; SCHÖNGART, J. Wood growth of Tabebuia barbata (E. Mey.) Sandwith (Bignoniaceae) and Vatairea guianensis Aubl. (Fabaceae) in Central Amazonian blackwater (igapó) and white-water (várzea) floodplain forests. Trees - Structure and Function, Berlin, v. 23, n. 1, p. 127-134, 2009.

FRITTS, H. C. Dendroclimatology and dendroecology. Quaternary Research, Cambridge, v. 1, n. 4, p. 419-449, 1971.

FRITTS, H. C.; LOFGREN, G. R.; GORDON, G. A. Variations in climate since 1602 as reconstructed from tree rings. Quaternary Research, Cambridge, v. 12, n. 1, p. 18-46, 1979.

FRITTS, H. C.; SWETNAM, T. W. Dendroecology: a tool for evaluating variations in past and present forest environments. Advances in Ecological Research, Amsterdam, v. 19, p. 111-188, 1989.

GARTNER, B. L. et al. Clues for dendrochronology from studies of wood structure and function. Dendrochronologia, Verona, v. 20, n. 1/2, p. 53-61, 2002.

HUGHES, M. K. Dendrochronology in climatology - the state of the art. Dendrochronologia, Verona, v. 20, n. 1/2, p. $95-116,2002$.

IBGE. Mapa de Biomas do Brasil. 2004. Disponível em: http://www.ibge.gov.br/home/presidencia/ noticias/21052004biomashtml.shtm. Acesso em: 24 jan. 2017.

LOPEZ, L.; VILLALBA, R. An assessment of Schinopsis brasiliensis Engler (Anacardiacea) for dendroclimatological applications in the tropical Cerrado and Chaco forests, Bolivia. Dendrochronologia, Verona, v. 40, p. 85-92, 2016.

LOPEZ, L.; VILLALBA, R.; BRAVO, F. Cumulative diameter growth and biological rotation age for seven tree species in the Cerrado biogeographical province of Bolivia. Forest Ecology and Management, Amsterdam, v. 292, 2013.

MARTINELLI, N. Climate from dendrochronology: Latest developments and results. Global and Planetary Change, Amsterdam, v. 40, n. 1/2, p. 129-139, 2004.

MENDIVELSO, H. A. et al. Agricultural and Forest Meteorology Time-dependent effects of climate and drought on tree growth in a Neotropical dry forest: Short-term tolerance vs long-term sensitivity. Agricultural and Forest Meteorology, Amsterdam, v. 188, p. 13-23, 2014.

NEUKOM, R.; GERGIS, J. Southern Hemisphere high-resolution palaeoclimate records of the last 2000 years. The Holocene, [S.1.], v. 22, n. 5, p. 501-524, 2011.

PAREDES-VILLANUEVA, K. et al. Rainfall and temperature variability in Bolivia derived from the tree-ring width of Amburana cearensis (Fr. Allem.) A.C. Smith. Dendrochronologia, Verona, v. 35, 2015.

PETERS, R. L. et al. Detecting long-term growth trends using tree rings : a critical evaluation of methods.

Global Change Biology, Urbana, v. 21, p. 2040-2054, 2015.

SOARES, L. C. Limites meridionais e orientais da área de ocorrência da floresta amazônica em território brasileiro. Revista Brasileira de Geografia, Rio de Janeiro, n. 1, 1953.

SCHÖNGART, J. et al. Phenology and stem growth periodicity of tree species in Amazonian floodplain forests. Journal of Tropical Ecology, Cambridge, v. 18, p. 581-597, 2002.

SCHÖNGART, J. et al. Teleconnection between tree growth in the Amazonian floodplains and the El Niño - Southern Oscillation effect. Global Change Biology, Urbana, v. 10, p. 683-692, 2004. 
SCHÖNGART, J. et al. Wood growth patterns of Macrolobium acaciifolium (Benth.) Benth. (Fabaceae ) in Amazonian black-water and white-water floodplain forests. Oecologia, New York, v. 145, p. 454-461, 2005.

SCHÖNGART, J.; JUNK, W. J. Forecasting the flood-pulse in Central Amazonia by ENSO-indices. Journal of Hydrology, Amsterdam, v. 335, n. 1/2, p. 124-132, 2007.

SHEPPARD, P. R. Dendroclimatology: extracting climate from trees. WIREs Climate Change, [S.1.], v. 1, n. 3, p. 343-352, 2010.

SOLIZ-GAMBOA, C. C. et al. Evaluating the annual nature of juvenile rings in Bolivian tropical rainforest trees. Trees - Structure and Function, Berlin, v. 25, n. 1, p. 17-27, 2011.

TARELKIN, Y. et al. Growth-ring distinctness and boundary anatomy variability in tropical trees. IAWA Journal, Leiden, v. 37, n. 2, p. 275-294, 2016.

VLAM, M. et al. Understanding recruitment failure in tropical tree species : insights from a tree-ring study. Forest Ecology and Management, Amsterdam, v. 312, p. 108-116, 2014.

WILES, G. C.; CALKIN, P. E.; JACOBY, G. C. Tree-ring analysis and Quaternary geology: principles and recent applications. Geomorphology, Amsterdam, v. 16, n. 3, p. 259-272, 1996.

WIMMER, R. Wood anatomical features in tree-rings as indicators of environmental change. Dendrochronologia, Verona, v. 20, n. 1, p. 21-36, 2002.

ZUIDEMA, P. A. et al. Tropical forests and global change: filling knowledge gaps. Trends in Plant Science, Amsterdam, v. 18, n. 8, 2013. 